\title{
Clinical Characteristics and Prevalence of Comorbidities according to Metformin Use in Korean Patients with Type 2 Diabetes
}

\author{
Sang Ouk Chin ${ }^{D},{ }^{1}$ In Gyoon Ha, ${ }^{1}$ Sang Youl Rhee ${ }^{D},{ }^{1}$ Su Jin Jeong, ${ }^{2}$ Suk Chon, ${ }^{1}$ \\ Sung Hoon Kim, ${ }^{3}$ Kyu Jeung Ahn, ${ }^{1}$ Sei Hyun Baik, ${ }^{4}$ Yongsoo Park, ${ }^{5}$ Moon Suk Nam, ${ }^{6}$ \\ Kwan Woo Lee $\mathbb{D}^{7}{ }^{7}$ and Jeong Taek Woo ${ }^{1}{ }^{1}$ \\ ${ }^{1}$ Department of Endocrinology and Metabolism, College of Medicine, Kyung Hee University, Seoul, Republic of Korea \\ ${ }^{2}$ Statistics Support Department, Kyung Hee University, Medical Center Science Research Institute, Seoul, Republic of Korea \\ ${ }^{3}$ Division of Endocrinology and Metabolism, Department of Medicine, Cheil General Hospital and Women's Healthcare Center, \\ College of Medicine, Dankook University, Yongin, Republic of Korea \\ ${ }^{4}$ Division of Endocrinology and Metabolism, Department of Internal Medicine, College of Medicine, Korea University, Seoul, \\ Republic of Korea \\ ${ }^{5}$ Department of Internal Medicine, College of Medicine, Hanyang University, Seoul, Republic of Korea \\ ${ }^{6}$ Department of Internal Medicine, College of Medicine, Inha University, Incheon, Republic of Korea \\ ${ }^{7}$ Department of Endocrinology and Metabolism, College of Medicine, Ajou University, Suwon, Republic of Korea
}

Correspondence should be addressed to Jeong Taek Woo; jtwoo@khu.ac.kr

Received 5 March 2020; Revised 15 June 2020; Accepted 30 June 2020; Published 24 July 2020

Academic Editor: Giuseppe Reimondo

Copyright ( $\odot 2020$ Sang Ouk Chin et al. This is an open access article distributed under the Creative Commons Attribution License, which permits unrestricted use, distribution, and reproduction in any medium, provided the original work is properly cited.

Background/Aims. This study was designed to identify the clinical characteristics of Korean patients with type 2 diabetes according to metformin use. Methods. This cross-sectional study based on the Korean National Diabetes Program 2 registry used its baseline clinical data collected from seven participating university hospitals in Korea. Patients with no significant changes in their oral hypoglycemic agents and no diabetes-related complications within the year prior to participation were enrolled. Patients' clinical characteristics according to metformin use were analyzed. Results. Among 858 subjects included in the analyses, 706 were metformin users and 152 were nonmetformin users. Metformin users were significantly younger and had higher and glycated hemoglobin with significantly lower rates of accompanying microvascular complications such as retinopathy, cataracts, overt proteinuria, renal insufficiency, and peripheral neuropathy than nonusers. Meanwhile, there was a significantly lower prevalence of malignancy and depression among metformin users. These associations remained significant in multivariate analyses. The prevalence rate of macrovascular complications was not significantly different between the two groups. Conclusions. There were significant differences with respect to clinical characteristics and comorbidity prevalence according to metformin use among Korean type 2 diabetes patients. Long-term follow-up of these patients is necessary to observe how this difference will affect clinical outcomes for these patients.

\section{Introduction}

The prevalence of type 2 diabetes in Korea is reported to be $12.6 \%$ in adults over age 30, according to the 2011 Korea National Health and Nutritional Examination Survey, which was based on a nationwide cross-sectional health surveillance program [1]. With its increasing prevalence, the medical care cost and the acute and chronic complication rates for diabetes should also likely increase.

Many clinical guidelines and consensus statements currently recommend metformin in combination with lifestyle management as the first treatment option for patients with newly diagnosed type 2 diabetes patients, if not contraindicated $[2,3]$. Metformin has been reported to offer 
the additional benefit of preventing cardiovascular diseases (CVD) [4], although this result was disputed in a recent meta-analysis [5]. Since metformin is eliminated by the kidneys [6], traditionally it has not been recommended for patients with moderate-to-severe renal insufficiency due to the possibility of lactic acidosis [7]. However, a recent study and meta-analysis demonstrated that metformin can be safely used in patients with mild-to-moderate chronic kidney disease, showing a similar incidence rate for lactic acidosis regardless of renal function $[8,9]$, which expands the potential range of patients that can be helped by metformin [2]. In addition to renal insufficiency, guidelines indicate other conditions in which metformin should not be used when treating patients with diabetes.

This means, despite the core role of metformin as a firstline treatment option as recommended in many guidelines, it is very common to avoid metformin and to prescribe other oral hypoglycemic agents when treating patients. Therefore, an investigation of patients' characteristics and their accompanying morbidities according to metformin use would help us to identify the unique features of two groups, which may enable us to improve our ability to provide individualized and effective treatment plans as well as to predict prognosis. Thus, this study was aimed to compare patient characteristics according to metformin use among the KNDP2 cohort study, which is a representative cohort of patients with diabetes in Korea.

\section{Methods}

2.1. KNDP2 Cohort Study. The Korean National Diabetes Program 2 (KNDP2) cohort study was a follow-up study of the KNDP study. The KNDP study was conducted from 2006 to 2011 and used a prospective, multicenter, observational design. Details of the study were described previously [10]. Using an identical design to evaluate Korean patients with type 2 diabetes, the KNDP2 cohort study aimed to establish clinical research infrastructure encompassing local clinics and university hospitals that enabled investigators to collect and analyze clinical data from patients with type 2 diabetes. Participating hospitals included Kyung Hee University Hospital at Gangdong, Cheil General Hospital and Women's Healthcare Center, Korea University Guro Hospital, Hanyang University Guri Hospital, Inha University Hospital, and Ajou University Hospital.

2.2. Subjects and Study Design. We conducted a cross-sectional study using KNDP2 registry data collected from the seven participating university hospitals. From May 2014 to March 2015, 858 patients with type 2 diabetes between ages 20 and 80 years with no diabetes-related acute or chronic complications within three months prior to KNDP2 enrollment were recruited into our study. Clinical data, including blood and urine samples, were collected via an electronic case report form (eCRF) from each participant after obtaining informed consent. The eCRF included critical clinical items, as recommended in the guidelines published by the American Diabetes Association and the Korean
Diabetes Association (KDA) [11, 12], and was developed after review and comments from investigators who participated in the KNDP2 study. The eCRF items were also endorsed by an expert group from the KDA, as described previously [13]. Based on this eCRF, common data elements (CDEs) and standard operating procedures (SOPs) were developed to secure standardized clinical and biochemical data and to establish practical action plans.

The eCRF includes the following information collected from interviews conducted during cohort recruitment and from medical record reviews: age, anthropometrics, and duration of diabetes comorbidities including hypertension, dyslipidemia, cancer, autoimmune diseases, tuberculosis, depression, periodontal disease, and macro-/microvascular complications. Medications included in the analysis were defined when satisfying the following criteria: oral hypoglycemic agents/insulin, antihypertensive drugs, or statins with a prescription history of $>2$ months in length and within 3 months of recruitment. Laboratory data were also collected at recruitment and included in the eCRF: fasting blood glucose, glycated hemoglobin A1c (HbAlc), total cholesterol (T-chol), triglycerides (TG), low-density lipoprotein cholesterol (LDL-C), high-density lipoprotein cholesterol (HDL-C), blood urea nitrogen (BUN), serum creatinine $(\mathrm{Cr})$, and urine microalbumin. Fasting blood glucose levels were measured using the hexokinase method after blood samples were centrifuged. HbAlc levels were determined using the high-performance liquid chromatography method that received National Glycohemoglobin Standardization Program certification. T-chol, TG, HDL-C, LDL-C, BUN, and Cr were analyzed using an automated analyzer that received a certification of quality control from the Korean Society for Laboratory Medicine. These clinical characteristics of enrolled patients from the eCRF were cross-sectionally collected and compared according to metformin use at the time of data collection.

2.3. Statistics. Patients were stratified by metformin use, and their clinical characteristics were analyzed. Descriptive values are expressed as means and standard deviations, and Student's $t$-test and the chi-square test were used to evaluate statistical significance. $P$ values $<0.05$ were considered significant. Logistic regression analysis was used to estimate correlations between the two groups. Univariate logistic regression was performed to estimate correlations for each variable and disease, and multiple logistic regression was performed to adjust for confounding variables, such as age, sex, and others (Figure 1 for the detailed explanation). Statistical analyzes were performed by a statistician at the Kyung Hee University Medical Science Research Institute. SAS software version 9.3 (SAS Institute Inc., Cary, NC, USA) was used to perform all statistical tests.

2.4. Ethics Statement. This study was approved by the Institutional Review Board of Kyung Hee University Hospital (KMC IRB 1428-03). Written informed consent was obtained from all participants. Data from this study are registered at the Clinical Research Information Service (CRIS, 


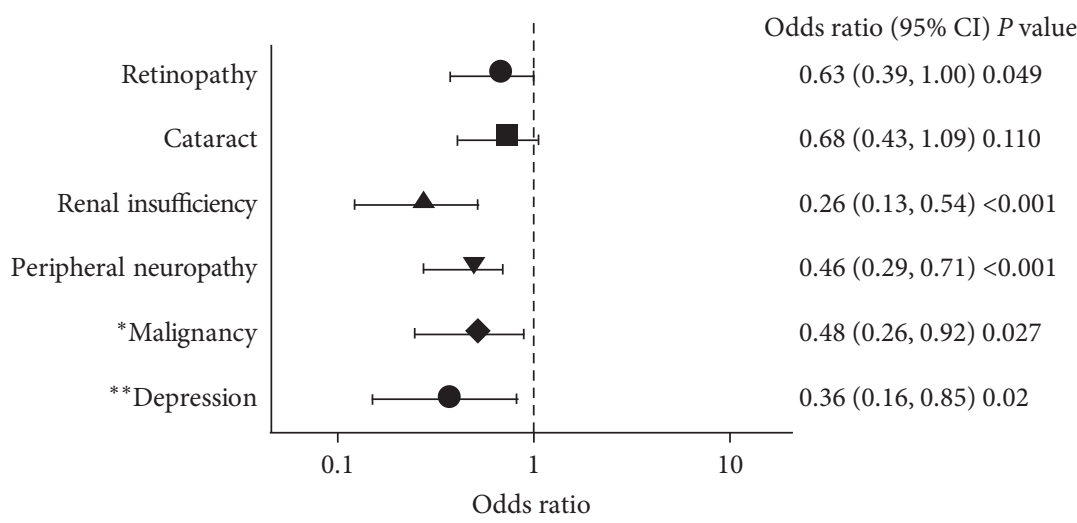

FIGURE 1: Multivariate logistic regression analysis to observe the association between metformin use and comorbidities adjusted by age, sex, BMI, HbA1c total cholesterol, history of hypertension/hyperlipidemia, medication for diabetes, antiplatelet medication, family history of comorbidity, smoking status, alcohol use, creatinine, and creatinine clearance. Malignancy adjusted by age, sex, family history of malignancy, smoking status, HbA1c, and diabetes duration. Depression adjusted by age, sex, history of stroke, family history of depression, and diabetes duration.

No. KCT0001232). The CRIS is a Korean national service connected to the International Clinical Trials Registry Platform of the World Health Organization.

\section{Results}

3.1. Baseline Characteristics. Table 1 shows baseline clinical characteristics of subjects according to metformin use. A total of 857 patients were enrolled in the study, of whom 706 $(82.3 \%)$ were taking metformin and 151 (17.7\%) were not. Other hypoglycemic agents used in either the metformin user group or nonuser group are listed in Table 2. There was no significant difference in sex proportions between the two groups. The mean age of the metformin user group was significantly younger, and they had shorter durations of diabetes than the nonuser group. The metformin users had significantly higher BMI, body weight, waist circumference, and hip circumference than metformin nonusers. Serum creatinine was significantly lower, and creatinine clearance was significantly higher in the metformin user group. The metformin user group also had significantly higher HbA1c and lower serum creatinine and LDL cholesterol levels.

3.2. Comparison of Comorbidities. There was a significant difference in comorbidities between the metformin user and nonuser groups (Table 3$)$. The prevalence of cancer $(n=38$ $(5.43 \%)$ in the metformin user group, and $n=17(11.3 \%)$ in the nonuser group) and depression $(n=18(2.57 \%)$ in the metformin user group, and $n=9(6.0 \%)$ in the nonuser group) was significantly lower in the metformin user group, according to chi-square analysis $(P=0.016$ for cancer, and $P=0.039$ for depression), and these results remained significant after adjusting for age and sex.

3.3. Comparison of Macro- and Microvascular Complications of Diabetes. Tables 4 and 5 show the prevalence of macroand microvascular complications of diabetes according to metformin use. While no significant difference was observed in macrovascular complications according to metformin use (Table 4), the prevalence of diabetic retinopathy, cataracts, overt proteinuria, renal insufficiency (estimated glomerular filtration rate $<60 \mathrm{~mL} / \mathrm{min} / 1.73 \mathrm{~m}^{2}$ ), and peripheral neuropathy was lower in the metformin user group (Table 5). To evaluate the independent association between metformin use and comorbidities, multivariate logistic regression analysis was performed with correction for sex, age, and other selected variables (Figure 1). Odds ratios for various comorbidities among metformin users compared with nonusers were as follows: retinopathy, $\mathrm{OR}=0.63$ (95\% CI: 0.39-1.00); renal insufficiency, $\mathrm{OR}=0.26 \quad(95 \% \mathrm{CI}$ : 0.13-0.54); peripheral neuropathy, $\mathrm{OR}=0.46$ (95\% CI: $0.29-0.71)$; malignancy, $\mathrm{OR}=0.48$ (95\% CI: $0.26-0.92)$; and 0.36 (95\% CI: 0.16-0.85).

\section{Discussion}

This cross-sectional study compared the clinical characteristics of patients with type 2 diabetes according to metformin use. Results indicate that the metformin user group had a significantly lower prevalence of accompanying microvascular complications and other comorbidities, such as cancer, compared with nonusers. However, there was no significant difference in prevalence of macrovascular complications between the metformin user and nonuser groups. These findings suggest that metformin nonusers have a higher risk of adverse outcomes for their prognosis, thus requiring more careful management of their diabetes.

Among our study patients, the metformin nonusers tended to be older but also had lower BMI and waist circumference, which may have been possibly due to relatively poor general condition associated with a longer duration of diabetes in these patients $(12.61 \pm 7.23$ vs $14.16 \pm 8.12$ years, $P=0.020$, Table 1$)$. Renal function was also significantly deteriorated among the nonuser group, and they had higher prevalence of microvascular complications such as proteinuria and renal insufficiency, which was reflected in their recent prescription patterns based on guidelines limiting 
TABLE 1: Difference of baseline characteristics of subjects according to the use of metformin.

\begin{tabular}{|c|c|c|c|}
\hline $\begin{array}{l}\text { Variable } \\
n\end{array}$ & $\begin{array}{c}\text { Metformin user } \\
706(82.3 \%)\end{array}$ & $\begin{array}{c}\text { Metformin nonuser } \\
152(17.7 \%)\end{array}$ & $P$ \\
\hline Sex, male/female & & & 0.210 \\
\hline Male, $n(\%)$ & $321(80.5 \%)$ & $78(19.5 \%)$ & \\
\hline Female, $n(\%)$ & $385(83.9 \%)$ & $74(16.1 \%)$ & \\
\hline Age, years old & $59.42 \pm 9.77$ & $63.16 \pm 9.87$ & $<0.001$ \\
\hline Duration of diabetes, years & $12.61 \pm 7.23$ & $14.16 \pm 8.12$ & 0.020 \\
\hline Body weight, kg & $66.75 \pm 11.68$ & $64.39 \pm 11.16$ & 0.023 \\
\hline Body mass index, $\mathrm{kg} / \mathrm{m}^{2}$ & $25.44 \pm 3.46$ & $24.67 \pm 3.23$ & 0.013 \\
\hline Waist circumference, $\mathrm{cm}$ & $89.02 \pm 8.73$ & $86.56 \pm 7.93$ & 0.004 \\
\hline Chest circumference, $\mathrm{cm}$ & $94.49 \pm 7.27$ & $92.69 \pm 7.43$ & 0.042 \\
\hline Hip circumference, $\mathrm{cm}$ & $95.2 \pm 6.55$ & $93.21 \pm 6.44$ & 0.002 \\
\hline Glycosylated hemoglobin, \% & $7.45 \pm 1.27$ & $7.1 \pm 1.37$ & 0.002 \\
\hline Fasting plasma glucose, $\mathrm{mg} / \mathrm{dL}$ & $150 \pm 49.62$ & $140.88 \pm 56.09$ & 0.063 \\
\hline Urine microalbumin, $\mu \mathrm{g} / \mathrm{mg} \mathrm{Cr}$ & $39.04 \pm 110.86$ & $50.46 \pm 141.85$ & 0.371 \\
\hline Serum creatinine, $\mathrm{mg} / \mathrm{dL}$ & $0.82 \pm 0.28$ & $1.11 \pm 1.35$ & 0.010 \\
\hline Creatinine clearance, $\mathrm{ml} / \mathrm{min}$ & $96.52 \pm 28.8$ & $84.5 \pm 30.98$ & $<0.001$ \\
\hline ALT, IU/L & $23.24 \pm 23.00$ & $24.29 \pm 15.63$ & 0.011 \\
\hline Total cholesterol, mg/dL & $161.71 \pm 32.86$ & $173.29 \pm 36.64$ & $<0.001$ \\
\hline Triglyceride, mg/dL & $146.92 \pm 87.65$ & $133.75 \pm 73.27$ & 0.056 \\
\hline LDL cholesterol, mg/dL & $89.73 \pm 28.84$ & $100.5 \pm 31.22$ & $<0.001$ \\
\hline HDL cholesterol, $\mathrm{mg} / \mathrm{dL}$ & $48.44 \pm 13.07$ & $51.51 \pm 15.65$ & 0.027 \\
\hline
\end{tabular}

ALT, alanine transaminase; LDL, low-density lipoprotein; and HDL, high-density lipoprotein. Results are expressed as mean \pm standard deviation or $n$ (\%). $P$ value for the $t$-test comparing the both groups with and without metformin.

TABLE 2: Difference in the use of medications according to the use of metformin.

\begin{tabular}{lccr}
\hline Variable & Metformin user & Metformin nonuser & $P$ \\
\hline Sulfonylurea & $312(44.2)$ & $48(31.6)$ & 0.004 \\
DPP-4 inhibitor & $279(39.5)$ & $36(23.7)$ & $9(5.9)$ \\
Meglitinide & $12(1.7)$ & $9(5.9)$ & 0.002 \\
Thiozolidinedione & $32(4.5)$ & $9(5.9)$ & 0.006 \\
$\alpha$-Glucosidase inhibitor & $11(1.6)$ & 0 & 0.008 \\
SGLT2 inhibitor & 0 & $11(7.2)$ & 1.000 \\
GLP-1 agonist & $2(0.3)$ & $27(17.8)$ & 0.008 \\
Rapid-acting insulin & $20(2.8)$ & $20(13.16)$ & 0.125 \\
Long-acting insulin & $92(13.0)$ & $60(8.5)$ & 0.073 \\
Premixed insulin & & & \\
\hline
\end{tabular}

Values are expressed as number (\%). $P$ value for the chi-square test comparing the groups with and without metformin. DPP-4, dipeptidyl peptidase-4; SGLT2, sodium-glucose transporter protein 2; and GLP-1, glucagon-like peptide-1.

TABLE 3: Difference of comorbidity according to the use of metformin.

\begin{tabular}{lccc}
\hline Variable & Metformin user & Metformin nonuser & $P$ \\
\hline Hypertension, $n(\%)$ & $417(59.32 \%)$ & $101(66.45 \%)$ & $0.17 \pm 9.97$ \\
Hypertension duration, years & $5.67 \pm 7.24$ & $97(63.82 \%)$ & 0.120 \\
Dyslipidemia, $n$ (\%) & $436(62.02 \%)$ & $5.50 \pm 6.21$ & 0.713 \\
Dyslipidemia duration, years & $4.44 \pm 5.22$ & $17(11.33 \%)$ & 0.053 \\
Cancer, $n$ (\%) & $38(5.43 \%)$ & $1.15 \pm 3.58$ & 0.016 \\
Cancer duration, years & $0.40 \pm 1.96$ & $2(1.33 \%)$ & 0.023 \\
Autoimmune disease, $n(\%)$ & $17(2.43 \%)$ & $9(6 \%)$ & 0.553 \\
Tuberculosis, $n(\%)$ & $21(3 \%)$ & $9(6 \%)$ & 0.086 \\
Depression, $n(\%)$ & $18(2.57 \%)$ & $26(17.33 \%)$ & 0.039 \\
Periodontal disease, $n(\%)$ & $72(10.29 \%)$ & 0.023 \\
\hline
\end{tabular}

Results are expressed as mean \pm standard deviation or $n(\%) . P$ value for the $t$-test or the chi-square test comparing both the groups with and without metformin.

metformin use in patients with impaired renal function $[2,14]$. The prevalence of diabetic retinopathy and neuropathy was also higher in the metformin nonuser group, which is consistent with previous findings of an association between diabetes duration and microvascular complications $[15,16]$. 
TABLE 4: Difference of macrovascular complication of diabetes according to the use of metformin.

\begin{tabular}{lccc}
\hline Variable & Metformin user & Metformin nonuser & $P$ \\
\hline Myocardial infarction, $n(\%)$ & $9(1.28 \%)$ & $2(1.32 \%)$ & 1.000 \\
Angina, $n(\%)$ & $55(7.58 \%)$ & $15(9.93 \%)$ & 0.414 \\
PTCA, $n(\%)$ & $7(1 \%)$ & $1(0.66 \%)$ & 1.000 \\
PCI with stent insertion, $n(\%)$ & $27(3.85 \%)$ & $8(5.3 \%)$ & 0.374 \\
CABG, $n(\%)$ & $4(0.57 \%)$ & $0(0 \%)$ & 1.000 \\
Peripheral artery disease, $n(\%)$ & $4(0.57 \%)$ & $0(0 \%)$ & 1.000 \\
\hline
\end{tabular}

PTCA, percutaneous transluminal coronary angioplasty; PCI, percutaneous coronary intervention; and CABG, coronary artery bypass graft surgery. Results are expressed as mean \pm standard deviation or $n(\%)$. $P$ value for the $t$-test or the chi-square test comparing the both groups with and without metformin.

TABLE 5: Difference of microvascular complication of diabetes according to the use of metformin.

\begin{tabular}{|c|c|c|c|}
\hline Variable & Metformin user & Metformin nonuser & $P$ \\
\hline Diabetic retinopathy, $n(\%)$ & $136(19.4 \%)$ & $44(29.14 \%)$ & 0.011 \\
\hline DR duration, years & $1.31 \pm 3.31$ & $2.33 \pm 4.30$ & 0.007 \\
\hline Photocoagulation, $n(\%)$ & $28(4.01 \%)$ & $14(9.27 \%)$ & 0.012 \\
\hline Duration, years & $0.29 \pm 1.68$ & $0.60 \pm 2.43$ & 0.131 \\
\hline Intravitreal injection, $n(\%)$ & $20(2.86 \%)$ & $12(7.95 \%)$ & 0.007 \\
\hline Duration, years & $0.17 \pm 1.07$ & $0.55 \pm 2.09$ & 0.028 \\
\hline Ophthalmic surgery, $n(\%)$ & $82(11.73 \%)$ & $30(19.87 \%)$ & 0.011 \\
\hline Duration, years & $0.78 \pm 2.79$ & $1.50 \pm 3.62$ & 0.023 \\
\hline Cataract, $n(\%)$ & $135(19.26 \%)$ & $50(33.11 \%)$ & $<0.001$ \\
\hline Duration, years & $1.05 \pm 2.99$ & $2.31 \pm 4.64$ & 0.002 \\
\hline Overt proteinuria, $n(\%)$ & $31(4.4 \%)$ & $16(10.53 \%)$ & 0.005 \\
\hline Duration, years & $0.25 \pm 1.33$ & $0.75 \pm 2.50$ & 0.018 \\
\hline Renal insufficiency, $n(\%)$ & $37(5.25 \%)$ & $29(19.08 \%)$ & $<0.001$ \\
\hline Duration, years & $0.36 \pm 1.68$ & $1.22 \pm 3.01$ & $<0.001$ \\
\hline Peripheral polyneuropathy, $n(\%)$ & $169(24.0 \%)$ & $63(41.45 \%)$ & $<0.001$ \\
\hline Duration, years & $1.48 \pm 3.07$ & $3.13 \pm 4.55$ & $<0.001$ \\
\hline
\end{tabular}

DR, diabetic retinopathy; PPN, peripheral polyneuropathy; Results are expressed as mean \pm standard deviation or $n$ (\%). $P$ value for $t$-test or chi-square test comparing both the groups with and without metformin.

Our study demonstrated that prevalence of cancer, depression, and periodontal disease was significantly higher in the metformin nonuser group. In addition, hypertension, hyperlipidemia, and tuberculosis were more prevalent in the nonuser group but not significantly. Patients with diabetes are known to be more likely to have a higher risk of accompanying a depressive condition, and people with depression are shown to have an increased risk of developing diabetes [17]. This bidirectional association between diabetes and depression would aggravate in those with poor glucose control [18]. This relationship is shown to be additive, which leads to a higher mortality than patients with diabetes or depression alone [19]. Patients with both diseases also are reported to have a higher risk to experience microvascular complications and periodontal disease $[20,21]$. As for cancer and diabetes, many epidemiologic studies have reported an elevated cancer risk among patients with diabetes [22-27]. In contrast, metformin is thought to have anticancer effects, according to previous in vitro studies [28-33]. Also, it has been reported that patients with diabetes accompanying depression or periodontal disease may expect further improvement by using metformin $[34,35]$; patients who do not use metformin to treat their diabetes will not experience these additional benefits that metformin users may be expected to experience, though it is not clear why some comorbidities were significantly more common among nonusers. Therefore, it is important to pay close attention to metformin nonusers by applying a more individualized pharmacological approach and emphasizing the importance of lifestyle modification for better glucose control and to prevent deterioration of accompanied diseases.

Previous studies which investigated the effects of metformin on cardiovascular outcomes have produced variable results. UKPDS34, which evaluated the effects of metformin on overweight patients with type 2 diabetes, found a reduction in the risk of nonfatal myocardial infarction [4]. However, two meta-analyses reported an increase in cardiovascular risk when metformin was used with sulfonylurea $[36,37]$, furthering the debate regarding metformin's effect on cardiovascular outcomes. On the contrary, a number of previous studies reported that sulfonylurea may increase the cardiovascular risk, especially when compared with metformin [38, 39]. The metformin users were shown to use sulfonylurea more frequently than nonusers (Table 2), which may have offset the cardiovascular benefit of metformin leading to the lack of difference in macrovascular complications between two groups (Table 4). Considering the practical difficulty in conducting a large-scale clinical study of metformin use, future long-term observation of our study population could yield more valuable data about the cardiovascular effects of metformin. 
A major limitation of this study is its cross-sectional design. Therefore, caution is necessary to avoid misinterpreting our results as having a causal relationship. Especially for depression, it is hard to draw the conclusion that use of metformin led to a lower prevalence of depression when considering our study design. Previous studies demonstrated the negative effect of depression, which can aggravate patient's glucose control [34] and the promising role of metformin on improvement of cognitive functions in an animal study by inducing anti-inflammatory effects [40] and promoting neurogenesis in hippocampus [41]. This study, by reviewing the real clinical data, indirectly confirmed the previously published results, in which metformin can demonstrate beneficial effects on patients with diabetes and depression. In addition, this study was based on carefully controlled multicenter cohort data that utilized CDEs and SOPs for robust analyses. It should be noted that few studies have investigated clinical characteristics of cohort participants according to metformin use. Also, the authors were not able to collect the data regarding the duration of metformin use, possible history of using metformin in the metformin nonuser group, and the reason for not using metformin in the nonuser group, as well as the duration after which the complications occurred. Last, sodium-glucose transport protein 2 (SGLT2) inhibitors and GLP-1 (glucagon-like peptide-1) agonists well-known for their benefits on the cardiovascular outcome according to large-scaled trials $[42,43]$ were shown to be rarely used in our KNDP2 cohort. It is because these two drugs had been newly introduced in Korea at the time of data collection and thus were not being commonly prescribed when compared with present time.

In conclusion, significant differences in clinical characteristics according to metformin use were observed among patients with diabetes. In particular, metformin nonusers had higher prevalence of accompanying comorbidities including cancers and microvascular complications such as retinopathy, peripheral neuropathy, and cataracts. We are currently investigating clinical outcomes among our cohort participants according to metformin use to observe the effect of metformin on future prognoses.

\section{Data Availability}

The data utilized and analyzed to support the findings of our study are included within the article.

\section{Conflicts of Interest}

The authors declare that they have no conflicts of interest.

\section{Authors' Contributions}

The authors Sang Ouk Chin and In Gyoon Ha contributed equally to this manuscript.

\section{References}

[1] S.-H. Ko, K. Han, Y.-H. Lee et al., "Past and current status of adult type 2 diabetes mellitus management in Korea: a national health insurance service database analysis," Diabetes \& Metabolism Journal, vol. 42, no. 2, pp. 93-100, 2018.

[2] M. J. Davies, D. A. D’Alessio, J. Fradkin et al., "Management of hyperglycaemia in type 2 diabetes, 2018. a consensus report by the American diabetes association (ADA) and the European association for the study of diabetes (EASD)," Diabetologia, vol. 61, no. 12, pp. 2461-2498, 2018.

[3] M. K. Kim, S.-H. Ko, B.-Y. Kim et al., "2019 Clinical practice guidelines for type 2 diabetes mellitus in Korea," Diabetes \& Metabolism Journal, vol. 43, no. 4, pp. 398-406, 2019.

[4] Group UPDSU, "Effect of intensive blood-glucose control with metformin on complications in overweight patients with type 2 diabetes (UKPDS 34). UK prospective diabetes study (UKPDS) group," The Lancet, vol. 352, no. 9131, pp. 854-865, 1998.

[5] S. J. Griffin, J. K. Leaver, and G. J. Irving, "Impact of metformin on cardiovascular disease: a meta-analysis of randomised trials among people with type 2 diabetes," Diabetologia, vol. 60, no. 9, pp. 1620-1629, 2017.

[6] G. G. Graham, J. Punt, M. Arora et al., "Clinical pharmacokinetics of metformin," Clinical Pharmacokinetics, vol. 50, no. 2, pp. 81-98, 2011.

[7] F. Kajbaf, P. Arnouts, M. De Broe, and J. D. Lalau, "Metformin therapy and kidney disease: a review of guidelines and proposals for metformin withdrawal around the world," Pharmacoepidemiology and Drug Safety, vol. 22, no. 10, pp. 1027-1035, 2013.

[8] S. E. Inzucchi, K. J. Lipska, H. Mayo, C. J. Bailey, and D. K. McGuire, "Metformin in patients with type 2 diabetes and kidney disease," Jama, vol. 312, no. 24, pp. 2668-2675, 2014.

[9] M. J. Crowley, C. J. Diamantidis, J. R. McDuffie et al., "Clinical outcomes of metformin use in populations with chronic kidney disease, congestive heart failure, or chronic liver disease," Annals of Internal Medicine, vol. 166, no. 3, pp. 191-200, 2017.

[10] S. Y. Rhee, S. Chon, M. K. Kwon et al., "Prevalence of chronic complications in Korean patients with type 2 diabetes mellitus based on the Korean national diabetes program," Diabetes \& Metabolism Journal, vol. 35, no. 5, pp. 504-512, 2011.

[11] S.-H. Ko, S.-R. Kim, D.-J. Kim et al., "2011 clinical practice guidelines for type 2 diabetes in Korea," Diabetes \& Metabolism Journal, vol. 35, no. 5, pp. 431-436, 2011.

[12] Association AD, "Standards of medical care in diabetes-2013," Diabetes Care, vol. 36, pp. S11-S66, 2013.

[13] S. Park, S. Y. Rhee, S. J. Jeong et al., "Features of long-standing Korean type 2 diabetes mellitus patients with diabetic retinopathy: a study based on standardized clinical data," Diabetes \& Metabolism Journal, vol. 41, no. 5, pp. 393-404, 2017.

[14] S.-H. Ko, K.-Y. Hur, S. Y. Rhee et al., "Antihyperglycemic agent therapy for adult patients with type 2 diabetes mellitus 2017: a position statement of the Korean diabetes association," Diabetes \& Metabolism Journal, vol. 41, no. 5, pp. 337-348, 2017.

[15] S. D. Solomon, E. Chew, E. J. Duh et al., "Diabetic retinopathy: a position statement by the American diabetes association," Diabetes Care, vol. 40, no. 3, pp. 412-418, 2017.

[16] P. Kempler, S. Tesfaye, N. Chaturvedi et al., "Autonomic neuropathy is associated with increased cardiovascular risk factors: the EURODIAB IDDM complications study," Diabetic Medicine, vol. 19, no. 11, pp. 900-909, 2002.

[17] V. Fiore, M. Marci, A. Poggi et al., "The association between diabetes and depression: a very disabling condition," Endocrine, vol. 48, no. 1, pp. 14-24, 2015. 
[18] S. H. Golden, M. Lazo, M. Carnethon et al., "Examining a bidirectional association between depressive symptoms and diabetes," Jama, vol. 299, no. 23, pp. 2751-2759, 2008.

[19] K. Semenkovich, M. E. Brown, D. M. Svrakic, and P. J. Lustman, "Depression in type 2 diabetes mellitus: prevalence, impact, and treatment," Drugs, vol. 75, no. 6, pp. 577-587, 2015.

[20] E. H. B. Lin, C. M. Rutter, W. Katon et al., "Depression and advanced complications of diabetes: a prospective cohort study," Diabetes Care, vol. 33, no. 2, pp. 264-269, 2010.

[21] H. P. C. Artese, A. M. Foz, S. Rabelo Mde et al., "Periodontal therapy and systemic inflammation in type 2 diabetes mellitus: a meta-analysis," PLoS One, vol. 10, no. 5, Article ID e0128344, 2015.

[22] I. Wolf, S. Sadetzki, R. Catane, A. Karasik, and B. Kaufman, "Diabetes mellitus and breast cancer," The Lancet Oncology, vol. 6, no. 2, pp. 103-111, 2005.

[23] A. G. Renehan and S. M. Shalet, "Diabetes, insulin therapy, and colorectal cancer," BMJ, vol. 330, no. 7491, pp. 551-552, 2005.

[24] S. S. Coughlin, E. E. Calle, L. R. Teras, J. Petrelli, and M. J. Thun, "Diabetes mellitus as a predictor of cancer mortality in a large cohort of US adults," American Journal of Epidemiology, vol. 159, no. 12, pp. 1160-1167, 2004.

[25] R. Huxley, A. Ansary-Moghaddam, A. Berrington De González, F. Barzi, and M. Woodward, "Type-II diabetes and pancreatic cancer: a meta-analysis of 36 studies," British Journal of Cancer, vol. 92, no. 11, pp. 2076-2083, 2005.

[26] S. C. Larsson, N. Orsini, K. Brismar, and A. Wolk, "Diabetes mellitus and risk of bladder cancer: a meta-analysis," Diabetologia, vol. 49, no. 12, pp. 2819-2823, 2006.

[27] B. D. Yi, Y. P. Bae, B. G. Kim et al., "The association between type 2 diabetes mellitus and colorectal cancer," Endocrinology and Metabolism, vol. 26, no. 2, pp. 126-132, 2011.

[28] C. Fan, Y. Wang, Z. Liu et al., "Metformin exerts anticancer effects through the inhibition of the Sonic hedgehog signaling pathway in breast cancer," International Journal of Molecular Medicine, vol. 36, no. 1, pp. 204-214, 2015.

[29] H.-S. Moon and C. S. Mantzoros, "Regulation of cell proliferation and malignant potential by irisin in endometrial, colon, thyroid and esophageal cancer cell lines," Metabolism, vol. 63, no. 2, pp. 188-193, 2014.

[30] S. Gou, P. Cui, X. Li, P. Shi, T. Liu, and C. Wang, "Low concentrations of metformin selectively inhibit CD133 (+) cell proliferation in pancreatic cancer and have anticancer action," PLoS One, vol. 8, no. 5, Article ID e63969, 2013.

[31] E. Karnevi, K. Said, R. Andersson, and A. H. Rosendahl, "Metformin-mediated growth inhibition involves suppression of the IGF-I receptor signalling pathway in human pancreatic cancer cells," BMC Cancer, vol. 13, no. 1, p. 235, 2013.

[32] K. Kato, J. Gong, H. Iwama et al., "The antidiabetic drug metformin inhibits gastric cancer cell proliferation in vitro and in vivo," Molecular Cancer Therapeutics, vol. 11, no. 3, pp. 549-560, 2012.

[33] H. Kato, Y. Sekine, Y. Furuya, Y. Miyazawa, H. Koike, and K. Suzuki, "Metformin inhibits the proliferation of human prostate cancer PC-3 cells via the downregulation of insulinlike growth factor 1 receptor," Biochemical and Biophysical Research Communications, vol. 461, no. 1, pp. 115-121, 2015.

[34] M. Guo, J. Mi, Q. M Jiang et al., "Metformin may produce antidepressant effects through improvement of cognitive function among depressed patients with diabetes mellitus," Clinical and Experimental Pharmacology \& Physiology, vol. 41, no. 9, pp. 650-656, 2014.
[35] C.-H. Tseng, "Metformin may reduce oral cancer risk in patients with type 2 diabetes," Oncotarget, vol. 7, no. 2, pp. 2000-2008, 2016.

[36] R. Boussageon, I. Supper, T. Bejan-Angoulvant et al., "Reappraisal of metformin efficacy in the treatment of type 2 diabetes: a meta-analysis of randomised controlled trials," PLoS Medicine, vol. 9, no. 4, Article ID e1001204, 2012.

[37] C. Lamanna, M. Monami, N. Marchionni, and E. Mannucci, "Effect of metformin on cardiovascular events and mortality: a meta-analysis of randomized clinical trials," Diabetes, Obesity and Metabolism, vol. 13, no. 3, pp. 221-228, 2011.

[38] S. H. Simpson, S. R. Majumdar, R. T. Tsuyuki, D. T. Eurich, and J. A. Johnson, "Dose-response relation between sulfonylurea drugs and mortality in type 2 diabetes mellitus: a population-based cohort study," Canadian Medical Association Journal, vol. 174, no. 2, pp. 169-174, 2006.

[39] C. L. Morgan, C. D. Poole, M. Evans, A. H. Barnett, S. JenkinsJones, and C. J. Currie, "What next after metformin? a retrospective evaluation of the outcome of second-line, glucoselowering therapies in people with type 2 diabetes," The Journal of Clinical Endocrinology \& Metabolism, vol. 97, no. 12, pp. 4605-4612, 2012.

[40] K. Labuzek, D. Suchy, B. Gabryel, A. Bielecka, S. Liber, and B. Okopien, "Quantification of metformin by the HPLC method in brain regions, cerebrospinal fluid and plasma of rats treated with lipopolysaccharide," Pharmacological Reports, vol. 62, no. 5, pp. 956-965, 2010.

[41] J. Wang, D. Gallagher, L. M. DeVito et al., "Metformin activates an atypical PKC-CBP pathway to promote neurogenesis and enhance spatial memory formation," Cell Stem Cell, vol. 11, no. 1, pp. 23-35, 2012.

[42] B. Zinman, C. Wanner, J. M. Lachin et al., "Empagliflozin, cardiovascular outcomes, and mortality in type 2 diabetes," New England Journal of Medicine, vol. 373, no. 22, pp. 2117-2128, 2015.

[43] S. P. Marso, G. H. Daniels, K. Brown-Frandsen et al., "Liraglutide and cardiovascular outcomes in type 2 diabetes," New England Journal of Medicine, vol. 375, no. 4, pp. 311-322, 2016. 